\title{
Un piccolo archivio di tavolette presargoniche provenienti da Umma-Zabala
}

Maria Elena MILONE *

Università di Messina

In questo articolo è presentata l'edizione in copia cuneiforme, traslitterazione e traduzione di un piccolo archivio di venti tavolette amministrative databili al periodo Early Dynastic III $b$ e provenienti dalla città-stato di Umma-Zabala. I testi in questione appartengono alla collezione del dr. Massimo Baldacci che ringrazio vivamente per il permesso di pubblicazione.

Per ciascuna tavoletta sono fornite informazioni circa le dimensioni, la cottura, il colore, la curvatura della superficie di recto e verso, delle autografie. L'attenzione rivolta a questi ed altri dettagli di ordine materiale, quali l'ordine di scrittura, la disposizione dei segni nelle caselle, il ductus è intesa ad agevolare eventuali comparazioni tra questi testi e le diverse centinaia di tavolette Early Dynastic III provenienti da Umma-Zabala attualmente suddivise tra varie collezioni e per grandissima parte in attesa di edizione ${ }^{1}$.

\footnotetext{
* maria.elena.milon@katamail.com

1 Per un elenco e una dettagliata descrizione dei documenti presargonici da datare, come l'archivio qui pubblicato, precedentemente al regno di Lugalzagesi, cf. M.A. Powell, HUCA 49 (1978) pp. 13-15: si tratta di $M V N 3,3 ; 87 ; 106$; NBC 10235 e forse Oberhuber, $S A K F$ 3; è inoltre possibile che qualcuno dei testi di dubbia attribuzione citati, come $B I N 8,19$, sia da ritenere antecedente al lungo regno del sovrano di Umma. Per quel che riguarda i materiali inediti, circa quattrocento
}

Sefarad 65 (2005) págs. 327-351

() CSIC

ISSN 037-0894 
Per quel che riguarda le traslitterazioni, si noti che i numeri cuneiformi sono stati resi in corsivo, i curviformi in carattere normale; nelle traduzioni sono state impiegate le seguenti equivalenze tra unità di misura: 1 gursaggal $\sim 240 \mathrm{l}, 1 \mathrm{iku} \sim 3600 \mathrm{~m}^{2}$ ovvero 0,36 ha.

1

\section{$34 \times 33 \times 15 \mathrm{~mm}$}

Cotta in tempi moderni; rossiccio; curvatura della superficie più apprezzabile sul verso.

r. i 1. 10x3+4 zíz gur-sag-gál

2. Má-gur 8 -si

3. $10 \times 4$ lá 3

r. ii 1. Ba-a

2. $10 \times 2+2 \mathrm{Ki}-\mathrm{NI}-\mathrm{mu}-\mathrm{zu}$ (anepigrafo)

v. i 1. gú-an-šè $60+10 \times 3+3$ zíz 'gur?-sag-`gál’

2. 1 (bùr) $11 / 2 \mathrm{iku}$

3. a-šà-SI

v. ii $\quad{ }^{\mathrm{d}}$ Dumu-zi Umma ${ }^{\mathrm{ki}}$

34 gursaggal (= 8.160 litri) di dicocco: Magursi; 37 (= 8.880 litri): Baa; 22 (= 5.280 litri): KiNImuzu. In totale, 93 gursaggal $(=22.320$ litri) di dicocco per 19,5 iku (= 7,02 ha. di terreno). Campo-SI di Dumuzi di Umma.

tavolette di svariate dimensioni e contenuti appartengono alla collezione della Cornell University (Ithaca, New York): le informazioni relative a questi testi, anche quelle riportate nel seguito dell'articolo, sono dovute alla generosità di D. I. Owen; un centinaio di tavolette -si tratta perlopiù di lunghi registri di terreni- sono parte della Schöyen Collection (Oslo): questi dati si devono alla cortesia di A. Westenholz; infine, una cinquantina di testi è di proprietà della Banca d'Italia (Roma) ed è edita in G. Visicato e Å. Westenholz, Testi Presargonici e Sargonici di varia provenienza delle Collezioni della Banca d'Italia (in corso di stampa). 
2

$32 \times 28 \times 14 \mathrm{~mm}$

Cotta in tempi moderni; rossiccio; curvatura della superficie più accentuata sul verso.
r. i $1 . \quad 6$ še gur-sag-gál
2. Má-gur - -si
r. ii $1 . \quad 7 \mathrm{Ba}-\mathrm{a}$
2. 4 Ki-NI-mu-zu
v. i 1. gú-an-šè $10 \times 2$ lá 3 gur-sag-gál
2. $4 \mathrm{iku}$
3. še giš-ra $\mathrm{u}_{4} 10+1$
v. ii GIŠGAL-dagal ${ }^{\mathrm{d}}$ Dumu-zi

6 gursaggal (= 1.440 litri) di orzo: Magursi; 7 (= 1.680 litri): Baa; 4 (= 980 litri): KiNImuzu. In totale, 17 gursaggal (= 4.100 litri) di orzo per $4 \mathrm{iku}\left(=1,44\right.$ ha. di terreno): cereali trebbiati nell' $11^{\mathrm{mo}}$ giorno. (Campo) ... largo di Dumuzi.

3

$33 \times 32 \times 15 \mathrm{~mm}$

Cotta in tempi moderni; rossiccio; curvatura della superficie più apprezzabile sul verso.
r. i 1. 4 zíz gur-sag-gál
2. Má-gur ${ }_{8}$-si
3. $6 \mathrm{Ba}-\mathrm{a}$
r. ii $1 . \quad 5 \mathrm{Ki}-\mathrm{NI}-\mathrm{mu}-\mathrm{zu}$
(anepigrafo) 
v. i 1. gú-an-šè $10+5$ zíz gur

2. $3 \mathrm{iku}$

3. še giš-ra $u_{4} 10+1$

4. a-šà-SI

v. ii ${ }^{\mathrm{d}}$ Dumu-zi Umma ${ }^{\mathrm{ki}}$

4 gursaggal (= 960 litri) di dicocco: Magursi; 6 (= 1.440 litri): Baa; 5 (= 1.200 litri): Kinimuzu. In totale, 15 gur (= 3.600 litri) di dicocco per $3 \mathrm{iku}\left(=1,08\right.$ ha. di terreno): cereali trebbiati nell' $11^{\mathrm{mo}}$ giorno. Campo-SI di Dumuzi di Umma.

4

$35 \times 32 \times 12 \mathrm{~mm}$

Cotta in tempi moderni; rossiccio; la curvatura delle due facce è pressoché identica.

r. i 1. 10x2+2 zíz gur-sag-gál

2. Ba-a (sopra cancellatura di Má-gur ${ }_{8}$-si)

3. $10 \mathrm{Ki}-\mathrm{NI}-\mathrm{mu}-\mathrm{zu}$

r. ii 1. $\quad[10+2]+2$ Má-gur $_{8}$-si (anepigrafo)

v. i 1. gú-an-šè $10 x 4+6$ zíz gur

2. 1 (eše) $1 \mathrm{iku}$

3. še giš-ra $u_{4} 10 \times 2$ lá 3 a-šà-SI

v. ii $\quad{ }^{\mathrm{d}}$ Dumu-zi Umma ${ }^{\mathrm{ki}}$

22 gursaggal (= 5.280 litri) di dicocco: Baa; 10 (= 2.400 litri): KiNImuzu; 14 (= 5.280 litri): Magursi. In totale, 46 (= 11.040 litri) di dicocco per $7 \mathrm{iku}\left(=2,52\right.$ ha. di terreno): cereali trebbiati nel $17^{\mathrm{mo}}$ giorno. Campo-SI di Dumuzi di Umma. 


\section{$26 \times 23 \times 12 \mathrm{~mm}$}

Cotta in tempi moderni; rossiccio; curvatura della superficie più apprezzabile sul verso.
r. i 1. 10 lá 1 še gur-sag-gál
2. Má-gur $r_{8}$-si
r. ii 1. $6+[1] \mathrm{Ki}-\mathrm{NI}-\mathrm{mu}-\mathrm{zu}$
2. 10 lá $1 \mathrm{Ba}-\mathrm{a}$
v. i 1. gú-an-šè $10 \times 2+5$ še gur
2. $4 \mathrm{iku}$
3. še giš-ra $\mathrm{u}_{4} 10 \times 2+2$
v. ii GIŠGAL-dagal ${ }^{\mathrm{d} D u m u-z i}$

9 gursaggal (= 2.160 litri di orzo): Magursi; 7 (= 1.680 litri): KiNImuzu; 9 (= 2.160 litri): Baa. In totale, 25 gur (=6.000 litri) di orzo per $4 \mathrm{iku}\left(=1,44\right.$ ha. di terreno): cereali trebbiati nel $22^{\mathrm{mo}}$ giorno. (Campo) ... largo di Dumuzi.

\section{6}

$29 \times 26 \times 13 \mathrm{~mm}$

Cotta in tempi moderni; rossiccio; curvatura della superficie più accentuata sul verso.
r. i $1 . \quad 4$ še gur-sag-gál
2. Má-gur ${ }_{8}-\mathrm{si}$
r. ii $1 . \quad 2 \mathrm{Ba}-\mathrm{a}$
2. $2 \mathrm{Ki}-\mathrm{NI}-\mathrm{mu}-\mathrm{zu}$
v. i $1 . \quad$ gú-an-šè 8 še gur-sag
2. $21 / 2 \mathrm{iku}$
3. še giš-ra $\mathrm{u}_{4} 10 \times 2+5$
v. ii GIŠGAL-dagal ${ }^{\mathrm{d} D u m u-z i}$ 
4 gursaggal (= 960 litri) di orzo: Magur-si; 2 (= 480 litri): Ba-a; 2 (= 480 litri): KiNImuzu. In totale, 8 (= 1.920 litri) di orzo per 2,5 iku (= 0,9 ha. di terreno): cereali trebbiati nel $25^{\mathrm{mo}}$ giorno. (Campo) $\ldots$ largo di Dumuzi.

7

$31 \times 28 \times 14 \mathrm{~mm}$

Cotta in tempi moderni; rossiccio; curvatura della superficie più apprezzabile sul verso.

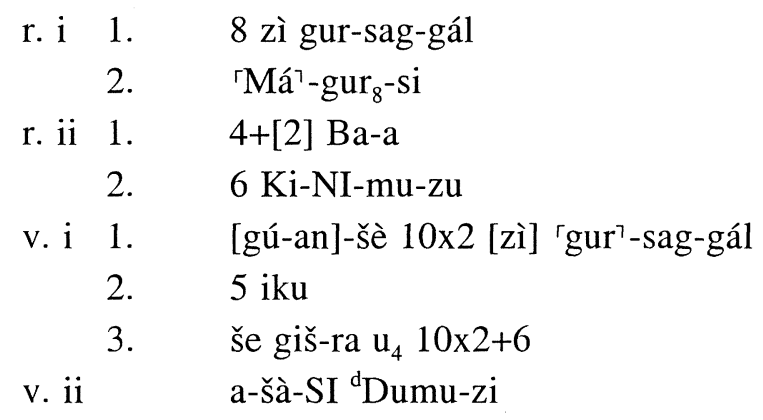

8 gursaggal (= 1.920 litri) di farina: Magursi; 6 (= 1.440 litri): Baa; 6 (= 1.440 litri): KiNImuzu. In totale, 20 (= 4.800 litri) di farina per $5 \mathrm{iku}$ (=1,8 ha. di terreno): cereali trebbiati nel $26^{\mathrm{mo}}$ giorno. Campo-SI di Dumuzi.

8

$28 \times 23 \times 16 \mathrm{~mm}$

Cotta in tempi moderni; rossiccio; curvatura della superficie lievemente più apprezzabile sul verso.

r. i $\quad 1 . \quad 8$ še gur-sag-gál

2. Má-gur ${ }_{8}$-si 


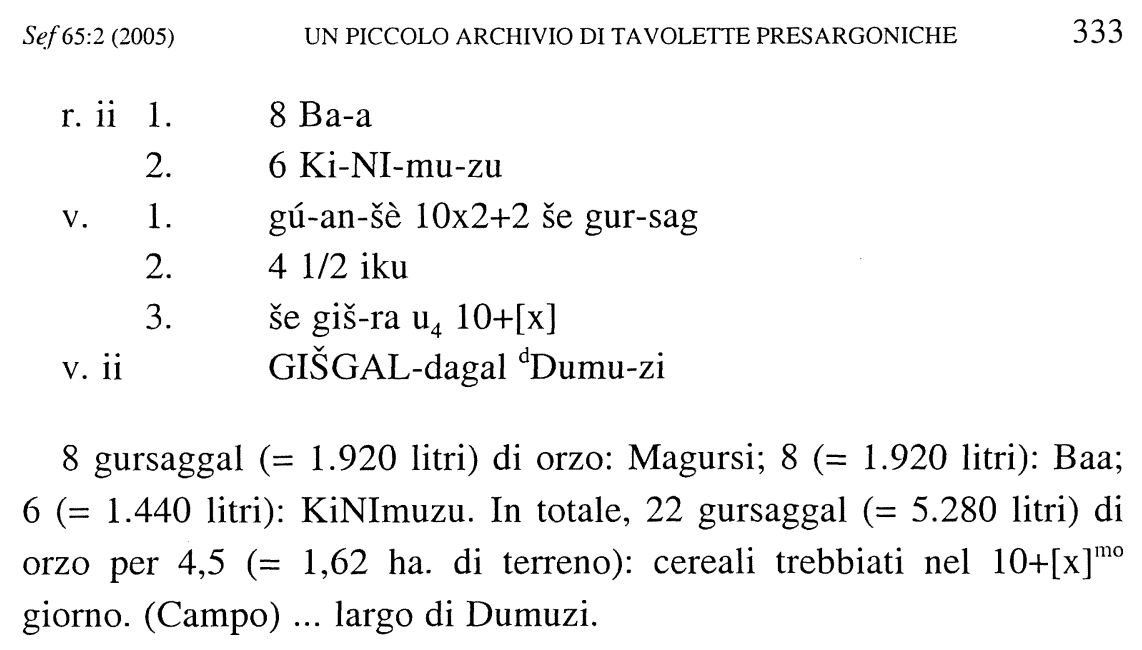

r. ii $1 . \quad 8 \mathrm{Ba}-\mathrm{a}$

2. $6 \mathrm{Ki}-\mathrm{NI}-\mathrm{mu}-\mathrm{zu}$

v. 1. gú-an-šè $10 \times 2+2$ še gur-sag

2. $41 / 2 \mathrm{iku}$

3. še giš-ra $\mathrm{u}_{4} 10+[\mathrm{x}]$

v. ii GIŠGAL-dagal ${ }^{\mathrm{d}}$ Dumu-zi

8 gursaggal (= 1.920 litri) di orzo: Magursi; 8 (= 1.920 litri): Baa; 6 (= 1.440 litri): KiNImuzu. In totale, 22 gursaggal (= 5.280 litri $) \mathrm{di}$ orzo per $4,5\left(=1,62\right.$ ha. di terreno): cereali trebbiati nel $10+[\mathrm{x}]^{\mathrm{mo}}$ giorno. (Campo) ... largo di Dumuzi.

9

$37 \times 34 \times 13 \mathrm{~mm}$

Cotta in tempi moderni; rossiccio; curvatura della superficie più accentuata sul verso.
r. i 1. [10] lá 1 zíz gur-sag-gál
2. Má-gur ${ }_{8}$-si
3. $10+3 \mathrm{Ba}-\mathrm{a}$
r. ii 1. $10 \mathrm{Ki}-\mathrm{NI}-\mathrm{mu}-\mathrm{zu}$ (anepigrafo)
v. i 1. gú-an-šè $10 \times 3+2$ zíz gur-sag-gál
2. $41 / 2 \mathrm{iku}$
3. še giš-ra $\mathrm{u}_{4} 10 \times 2+2$
v. ii $\quad$ a-šà ${ }^{\top}-S I{ }^{d}$ Dumu-zi Ummak ${ }^{k i} 7 \mathrm{mu}$

9 gursaggal (= 2.160 litri) di dicocco: Magursi; 13 (= 3.120 litri): Baa; 10 (= 2.400 litri) KiNImuzu. In totale, 32 gursaggal (= 7.680 litri) di dicocco per 4,5 iku $(=1,62$ ha. di terreno): cereale trebbiato nel $22^{\mathrm{mo}}$ giorno. Campo-SI di Dumuzi di Umma; $7^{\circ}$ anno. 
$37 \times 34 \times 15 \mathrm{~mm}$

Non cotta; rossiccio; curvatura della superficie lievemente più apprezzabile sul verso.

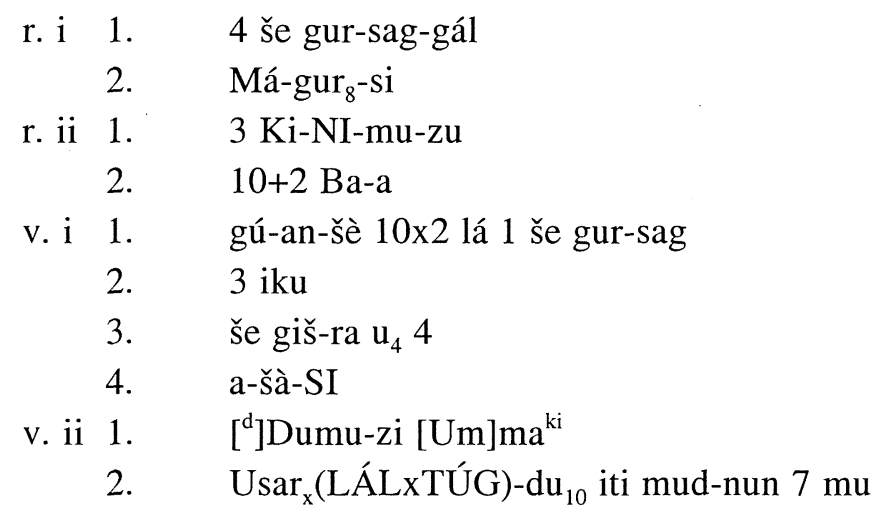

4 gursaggal (= 960 litri) di orzo: Magursi; 5 (= 720 litri): KiNImuzu; 12 (= 2.880 litri): Baa. In totale, 19 gursaggal (= 4.560 litri) di orzo per $3 \mathrm{iku}(=1,08$ ha. di terreno): cereali trebbiati nel $4^{\circ}$ giorno. Campo-SI di Dumuzi di Umma. (Periodo di) Usardu, mese di Mudnun, $7^{\circ}$ anno.

11

\section{$38 \times 34 \times 15 \mathrm{~mm}$}

Cotta in tempi moderni; rossiccio; curvatura della superficie lievemente più apprezzabile sul verso.

\begin{tabular}{|c|c|}
\hline i 1. & 4 še ‘gur? -sag-gál \\
\hline 2. & 7 zíz \\
\hline 3. & Má-gur - -si \\
\hline 4. & 5 še \\
\hline
\end{tabular}




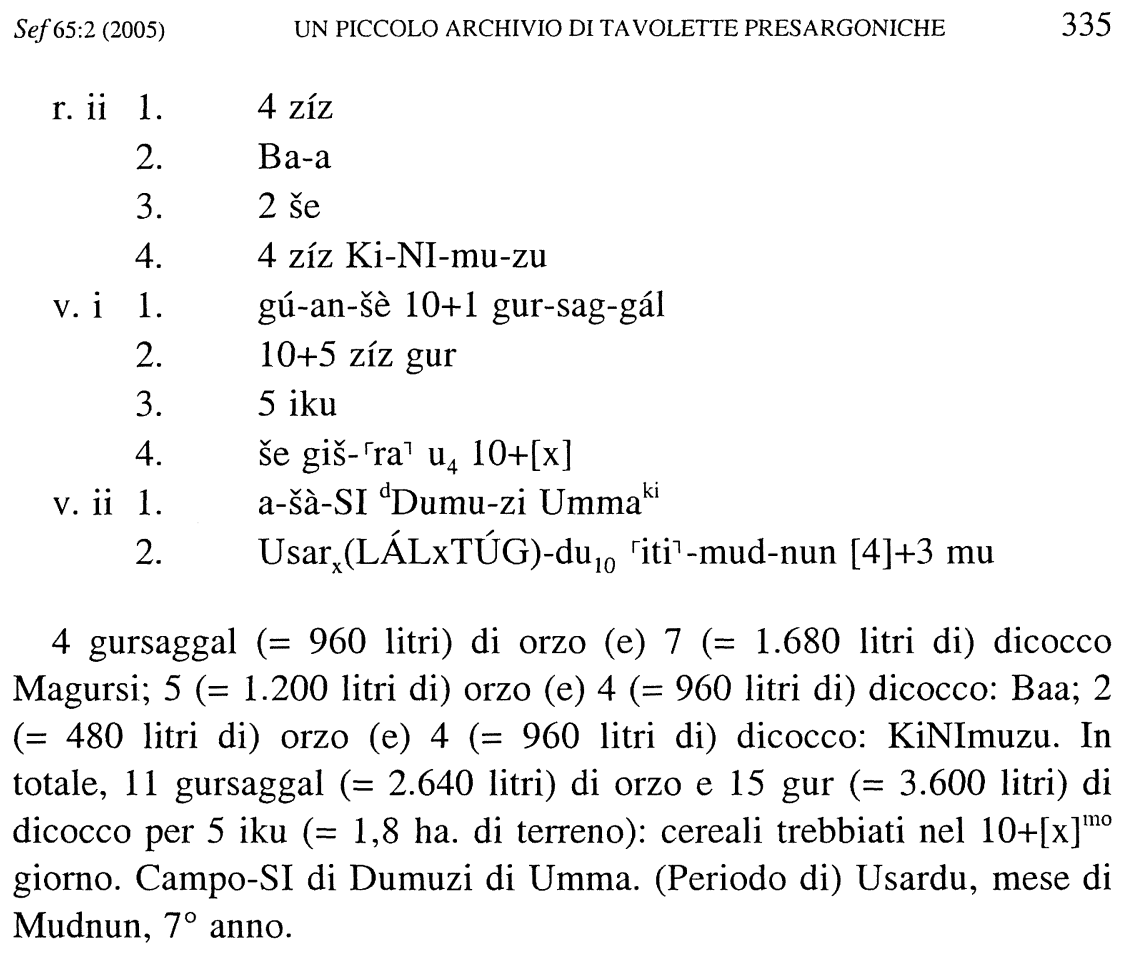

4 gursaggal (= 960 litri) di orzo (e) 7 (= 1.680 litri di) dicocco Magursi; 5 (= 1.200 litri di) orzo (e) 4 (= 960 litri di) dicocco: Baa; 2 (= 480 litri di) orzo (e) 4 (= 960 litri di) dicocco: KiNImuzu. In totale, 11 gursaggal (= 2.640 litri) di orzo e 15 gur $(=3.600$ litri $)$ di dicocco per $5 \mathrm{iku}\left(=1,8\right.$ ha. di terreno): cereali trebbiati nel $10+[\mathrm{x}]^{\mathrm{mo}}$ giorno. Campo-SI di Dumuzi di Umma. (Periodo di) Usardu, mese di Mudnun, $7^{\circ}$ anno.

12

$38 \times 33 \times 17 \mathrm{~mm}$.

Cotta in tempi moderni; rossiccio; curvatura della superficie più accentuata sul verso.

r. i 1. 10x3 lá 2 še gur še numun

2. 4 še $\mathrm{gu}_{4}-\mathrm{kú}$

3. É-kur-sikil du[b-sa]r

r. ii 1. ab-ta-sum-ma

2. $[\ldots]$

v. $\quad{ }^{\mathrm{d}}$ Inanna Zabala ${ }_{6}(\mathrm{AB} . \mathrm{MU} \check{\text { S̆ }})$

28 gur (= 6.720 litri) di orzo per semente (e) 4 (=960 litri) di orzo per l'alimentazione dei bovini: Ekursikil, lo scriba. Sono stati dati ... (Household templare di) Inanna di Zabala. 
13

$32 \times 28 \times 15 \mathrm{~mm}$

Cotta in tempi moderni; rossiccio; curvatura della superficie più accentuata sul recto.

r. i 1. $60 \times 4+10 \times 3$ lá 3 še gur

2. É-[ú]r-bi- ${ }^{\top} \mathrm{du}_{10}{ }^{\top}$

r. ii $1 . \quad 60 \times 5$ lá 3 še gur

2. ugula nu-bànda

3. $60 \times 5$ Ur-nàb

v. (anepigrafo)

267 gur (= 64.080 litri) di orzo: E-urbidu; 297 gur (= 71.280 litri): il responsabile dei capitani; 300 (= 72.000 litri): Ur-nab.

\section{4}

$26 \times 24 \times 14 \mathrm{~mm}$.

Cotta in tempi moderni; rossiccio; la curvatura delle due facce è pressoché identica.
r. i $1 . \quad 7.2$ še gu[r]
2. Šeš-pàd engar
r. ii ab-bar
v. (anepigrafo)

7 (gur) e 2 (bariga) (= 1.800 litri) di orzo: Šešpad, il fattore. Sono stati aggiunti.

\section{5}

\section{$27 \times 25 \times 13 \mathrm{~mm}$}

Cotta in tempi moderni; rossiccio; curvatura della superficie più accentuata sul recto. 


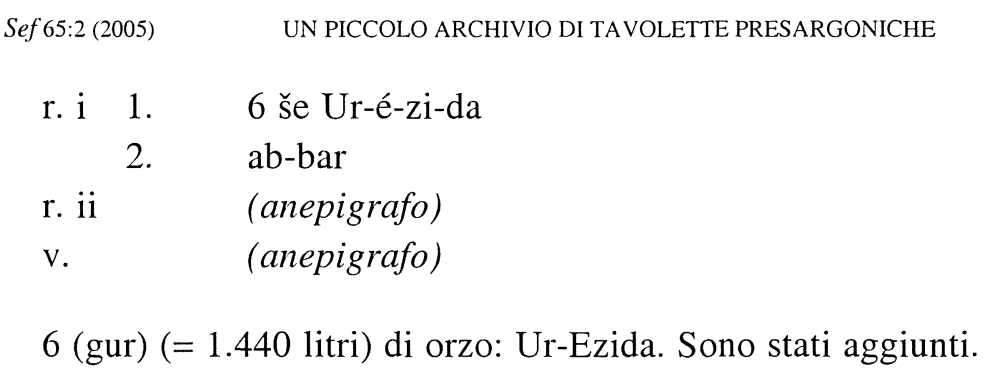

16

$21 \times 18 \times 12 \mathrm{~mm}$

Cotta in tempi moderni; rossiccio; curvatura della superficie più apprezzabile sul verso.

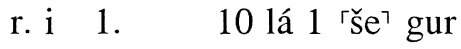
2. Lugal-DU
r. ii (anepigrafo)
v. $\quad$ tab-ba-am 6

9 gur (= 2.160 litri) di orzo: Lugal-DU. (La quantità di cereali) è stata radoppiata.

17

$27 \times 23 \times 13 \mathrm{~mm}$

Cotta in tempi moderni; rossiccio; curvatura della superficie lievemente più apprezzabile sul verso.
r. i $1 . \quad 0.0 .4$ še gur
2. Amar-é-sa
3. sum-ma
r. ii $\quad \mathrm{u}_{4} 1(/ / 10)$
v. (anepigrafo)
4 (bán) (= 40 litri) di orzo: Amar-Esa. Dati nel 1\%/10 1 mo giorno. 
$34 \times 29 \times 16 \mathrm{~mm}$

Cotta in tempi moderni; rossiccio; curvatura della superficie più apprezzabile sul recto.

r. i 1. $\quad\ulcorner 10 \times 4\urcorner$ še gur-[sag]-gál

2. Ur- ${ }^{\mathrm{I}} \mathrm{I} l d u m$

3. Ur- ${ }^{\mathrm{S}}$ Šákan(MUŠ)

r. ii $1 . \quad$ sum-ma

2. $\mathrm{u}_{4} 4$

3. Giš-gi-KI[D?]

v. (anepigrafo)

40 gursaggal (= 9.600 litri) di orzo: Ur-Ildum (e) Ur-Šakan. Dati. $4^{\circ}$ giorno. Canneto di ...

\section{9}

$32 \times 27 \times 15 \mathrm{~mm}$

Cotta in tempi moderni; rossiccio; la curvatura delle due facce è pressoché identica.
r. i $1 . \quad 1$ še gur
2. Ur- ${ }^{\mathrm{d}} \mathrm{Nin}-\mathrm{ild} u \mathrm{~m}$ sum-ma
r. ii $\quad u_{4} 5$
v. $\quad \mathrm{Umma}^{\mathrm{ki}}$
1 gur (= 240 litri) di orzo: Ur-Ninildum. Dati nel $5^{\circ}$ giorno. Umma. 


\section{0}

\section{$24 \times 21 \times 13 \mathrm{~mm}$}

Cotta in tempi moderni; rossiccio; curvatura della superficie più apprezzabile sul verso.

$$
\begin{array}{lll}
\text { r. i } & \text { 1. } & 2.2 \mathrm{u}_{4} 4 \\
& 2 . & 2.2 \mathrm{u}_{4} 5 \\
& 3 . & 2.2 \mathrm{u}_{4} 6 \\
\text { r. ii } & & \text { (anepigrafo) } \\
\text { v. } & & \text { (anepigrafo) }
\end{array}
$$

2 (gur) (e) 2 (bariga) (= 600 litri) nel $4^{\circ}$ giorno; 2 (gur) (e) 2 (bariga) (= 600 litri) nel $5^{\circ}$ giorno; 2 (gur) (e) 2 (bariga) (=600 litri) nel $6^{\circ}$ giorno.

\section{AlCUNE OSSERVAZIONI}

\section{La grafia}

Per la larghezza delle colonne, con la prima di norma più larga della seconda, e le tracce dello stilo lungo le linee divisorie verticali, sembra plausibile ricostruire l'ordine di scrittura delle nostre tavolette come segue: era dapprima redatto il testo della prima riga del recto e solo in seguito erano nell'ordine tracciate, con lo stilo, la linea divisoria verticale e quella orizzontale; la suddivisione della superficie in caselle avveniva, dunque, per mezzo dello stilo con il procedere della scrittura. Nelle tavolette scritte su ambedue le facce (nn. 1-11) il testo, inusualmente, dopo la rotazione prosegue nel verso sulla colonna di sinistra anziché su quella di destra ${ }^{2}$.

2 Sulla scrittura cuneiforme cf. in generale M. A. PowELL, «Three Problems in the History of Cuneiform Writing: Origins, Direction of Script, Literacy», Visible Language 15 (1981) pp. 419-440. 
Per quel che concerne il ductus, in alcuni testi la grafia appare più rozza, in altri più fine: questa difformità sembra da ricondurre all'impiego di stili a sezione molto assottigliata ovvero ben squadrata. La disposizione dei segni nelle caselle, infine, risulta non ben ponderata; a ogni modo, l'ordine dei segni è solitamente quello di lettura con l'eccezione del toponimo per Umma reso di norma con la sequenza grafemica GIŠ.KI.ÙH (cf. tavolette nn. 1, 3, 4, 8, 10, 11, 19).

\section{La datazione e la provenienza}

Le nostre tavolette, per un complesso di elementi di ordine interno ${ }^{3}$, sono databili al periodo Early Dynastic III b. Come esplicitamente indicato nei testi nn. 1-11 dalle date, dalla coerenza tematica, dalla prosopografia, esse sono state con ogni probabilità redatte in un lasso di tempo alquanto ridotto. Nello specifico, per la datazione delle tavolette 9-11 al settimo anno e la menzione in calce alle $\mathrm{nn} .10$ e 11 di Usardu, che da due testi della Cornell Collection sappiamo avere rivestito la carica di ensi di Umma ${ }^{4}$, è possibile datare le tavolette nn. 1-11 al settimo anno dell'ensiato di questo personaggio; sembrerebbe altresì plausibile datare l'intero archivio al medesimo periodo.

La provenienza dei nostri documenti dallo staterello di UmmaZabala può ritenersi certa per la menzione dei due toponimi (Umma nei testi nn. 1, 3, 4, 9, 10,11, 19 e Zabala nella tavoletta n. 12) e per quella di Usardu, ensi di Umma, ma anche per la presenza di antro-

3 Si tratta della forma molto arrotondata con una certa curvatura della superficie; della caratteristica suddivisione in colonne e ampie caselle; della paleografia per cui si notino in particolare le varianti di segni quali DU, SAG, ŠE, TUR e ZI; dell'ordine dei segni che normalmente coincide con quello di lettura; dell'uso della formula amministrativa gú-an-š̀̀.

4 Le tavolette in questione sono CUNES 00-08-005 (la sigla CUNES, impiegata qui e nel seguito dell'articolo, sta per Cornell University Near Eastern Studies) e 4810-043: la prima è un lungo riepilogo di assegnazioni di cereali e pani datato a Usar $_{\mathrm{x}}(\mathrm{LÁ} L x T U ́ \mathrm{G})-\mathrm{du}_{10}$ ensí Umma ${ }^{\mathrm{ki}}$; la seconda è un contratto di compravendita di una casa che reca la seguente formula di datazione: $\mathrm{u}_{4}$-ba Usar ${ }_{\mathrm{y}}$ (LÁLxDÚR)- $\mathrm{du}_{10}$ ensí Umma ${ }^{\mathrm{ki}}$ / Ama-bará-si sanga Zabala $(\mathrm{MÙS}$. AB) / 4 mu. 
ponimi quali Ur-Ildum (n. 18) e Ur-Ninildum (n. 19), i cui elementi teofori risultano particolarmente frequenti nell'onomastica di Umma, e per la paleografia.

\section{Il contenuto e la prosopografia}

Per quel che concerne il contenuto, il nostro archivio si presenta piuttosto omogeneo: la totalità dei testi riguarda infatti attività agricole e/o cereali, quali orzo, dicocco e derivati.

In particolare, le tavolette nn. 1-11 registrano il totale di cereali trebbiati in appezzamenti della household di Dumuzi sotto la responsabilità dei seguenti funzionari, elencati nella quasi totalità dei casi (otto su undici) nella medesima sequenza ${ }^{5}$ : Magursi, Baa e KiNImuzu. Nella tavola che segue sono riportati gli interessanti dati relativi al rapporto tra quantità di cereali trebbiati e l'estensione di terreno desumibili per ciascun testo, con indicazione del campo di riferimento:
N. Tav
Rapporto tra quantiı̀̀ di cereali
Campo
e estensione di terreno
n. 14,769 gur di dicocco per iku Campo-SI di Dumuzi
n. 24,25 gur di orzo per iku
(Campo)... largo di Dumuzi
n. 35 gur di dicocco per iku
Campo-SI di Dumuzi
n. $4 \quad 6,571$ gur di dicocco per iku
Campo-SI di Dumuzi
n. $5 \quad 6,25$ gur di orzo per iku
(Campo) ... largo di Dumuzi
n. 63,2 gur di orzo per iku
(Campo) ... largo di Dumuzi
n. 74 gur di farina per iku
Campo-SI di Dumuzi
n. 84,888 gur di orzo per iku
(Campo)... largo di Dumuzi
ก. 9
7,111 gur di dicocco per iku
Campo-SI di Dumuzi

\footnotetext{
5 I testi che presentano una serie differente sono la tavoletta n. 4 (Baa, KiNImuzu, Magursi), la 5 e la 10 (Magursi, KiNImuzu, Baa); è da notare che nel testo $\mathrm{n}$. 4 il nome del primo funzionario citato, Baa, appaia su cancellatura di Magursi.
} 

n. 10
6,333 gur di orzo per iku
Campo-SI di Dumuzi

n. 11

5,2 gur di orzo e dicocco per iku Campo-SI di Dumuzi

Quanto alla prosopografia, i paralleli più significativi riguardano la collezione Cornell ${ }^{6}$ : i tre funzionari su elencati figurano, infatti, in CUNES 00-08-058; Magursi e Baa in CUNES 00-10-009; Magursi e KiNImuzu in CUNES 01-10-008; il solo KiNImuzu in CUNES 0006-011 e 48-08-022.

Inoltre, in aggiunta alle succitate attestazioni di Usardu in CUNES 00-08-005 e 48-10-043, per quel che riguarda la tavoletta n. 13, abbiamo menzione di E-urbidu (r. i 2) in CUNES 00-08-59, 02-04$123,02-04-144,48-10-122$, di un capitano come sorvegliante (r. ii 2) in CUNES 00-08-035, di Ur-nab (r. ii 3) in CUNES 00-08-059, 0107-061, 02-04-144, 48-08-136; infine, per quel che riguarda rispettivamente i testi n. 18 e 19, Ur-Ildum appare in CUNES 00-06-009, 00-08-041, 01-02-027, 02-07-052, 48-07-91 e Ur-Ninildum in CUNES 00-06-044.

\section{INDICI}

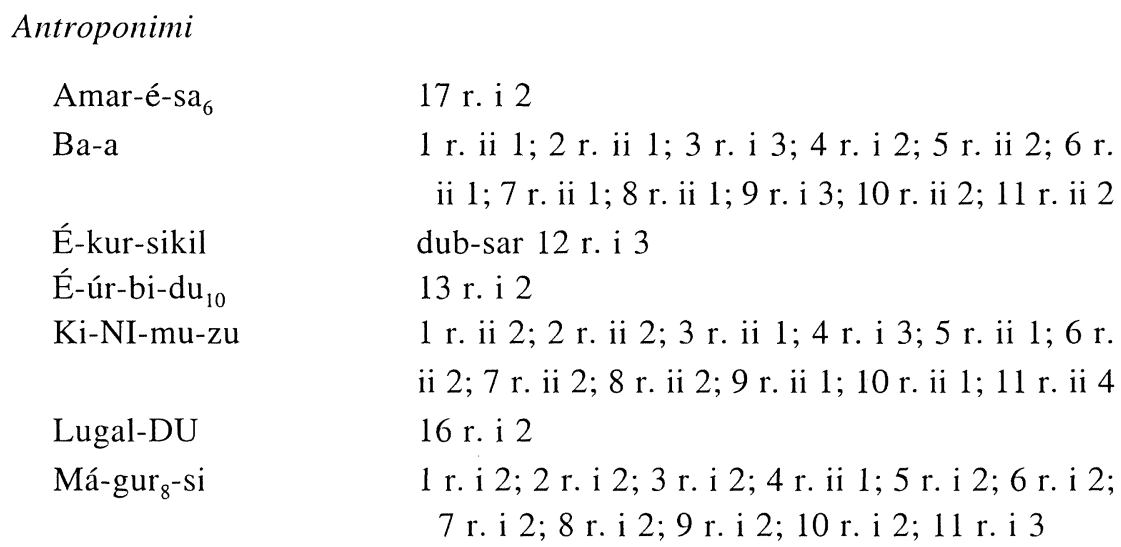

6 Si noti che i legami prosopografici di seguito evidenziati non hanno alcuna pretesa di esaustività: i testi della Cornell sono, infatti, a tutt'oggi inediti e le informazioni riportate si basano esclusivamente sui materiali di cui al momento è disponibile una traslitterazione. 


\begin{tabular}{|c|c|}
\hline \multirow{2}{*}{\multicolumn{2}{|c|}{ UN PICCOLO ARCHIVIO DI TAVOLETTE PRESARGONICHE }} \\
\hline & \\
\hline Šeš-pàd & engar 14 r. i 2 \\
\hline Ur-é-zi-da & 15 r. i 1 \\
\hline Ur- ${ }^{\mathrm{d}}$ Ildum & 18 r. i 2 \\
\hline Ur-nàb & 13 r. ii 3 \\
\hline Ur- ${ }^{\mathrm{d}} \mathrm{Nin}-\mathrm{illdum}$ & 18 r. i 2 \\
\hline Ur- ${ }^{\text {SSákan }}$ & 18 r. i 3 \\
\hline Usar $_{x}(\mathrm{LÁLxTÚG})-\mathrm{du}_{10}$ & 10 v. ii $2 ; 11$ v. ii 2 \\
\hline \multicolumn{2}{|l|}{ Nomi di professione } \\
\hline dub-sar & 12 r. i 3 \\
\hline engar & 14 r. i 2 \\
\hline ugula & nu-bànda 13 r. ii 2 \\
\hline \multicolumn{2}{|c|}{ Toponimi e centri agricolo-amministrativi } \\
\hline a-šà-SI d Dumu-zi & $\begin{array}{l}1 \text { v. i } 3 \text { - v. ii; } 3 \text { v. i } 4 \text { - v. ii; } 4 \text { v. i } 3 \text { - v. ii; } \\
7 \text { v. ii; } 9 \text { v. ii; } 10 \text { v. i } 4 \text { - v. ii } 1 ; 11 \text { v. ii } 1\end{array}$ \\
\hline Giš-gi-KI[D? $\left.{ }^{?}\right]$ & 18 v. ii 3 \\
\hline GIŠGAL-dagal d Dumu-zi & 2 v. ii; 5 v. ii; 6 v. ii; 8 v. ii \\
\hline${ }^{\mathrm{d}}$ Inanna & 12 v. (Household templare di) \\
\hline $\mathrm{Umma}^{\mathrm{ki}}$ & $\begin{array}{l}1 \text { v. ii; } 3 \text { v. ii; } 4 \text { v. ii; } 9 \text { v. ii; } 10 \text { v. ii } 1 ; 11 \text { v. } \\
\text { ii } 1 ; 19 \text { v. }\end{array}$ \\
\hline Zabala $_{6}$ & $12 \mathrm{v}$ \\
\hline
\end{tabular}




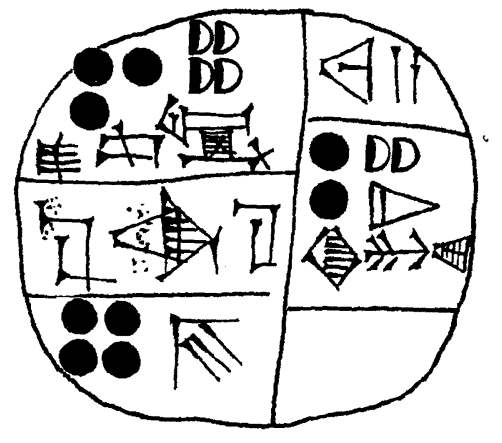

1
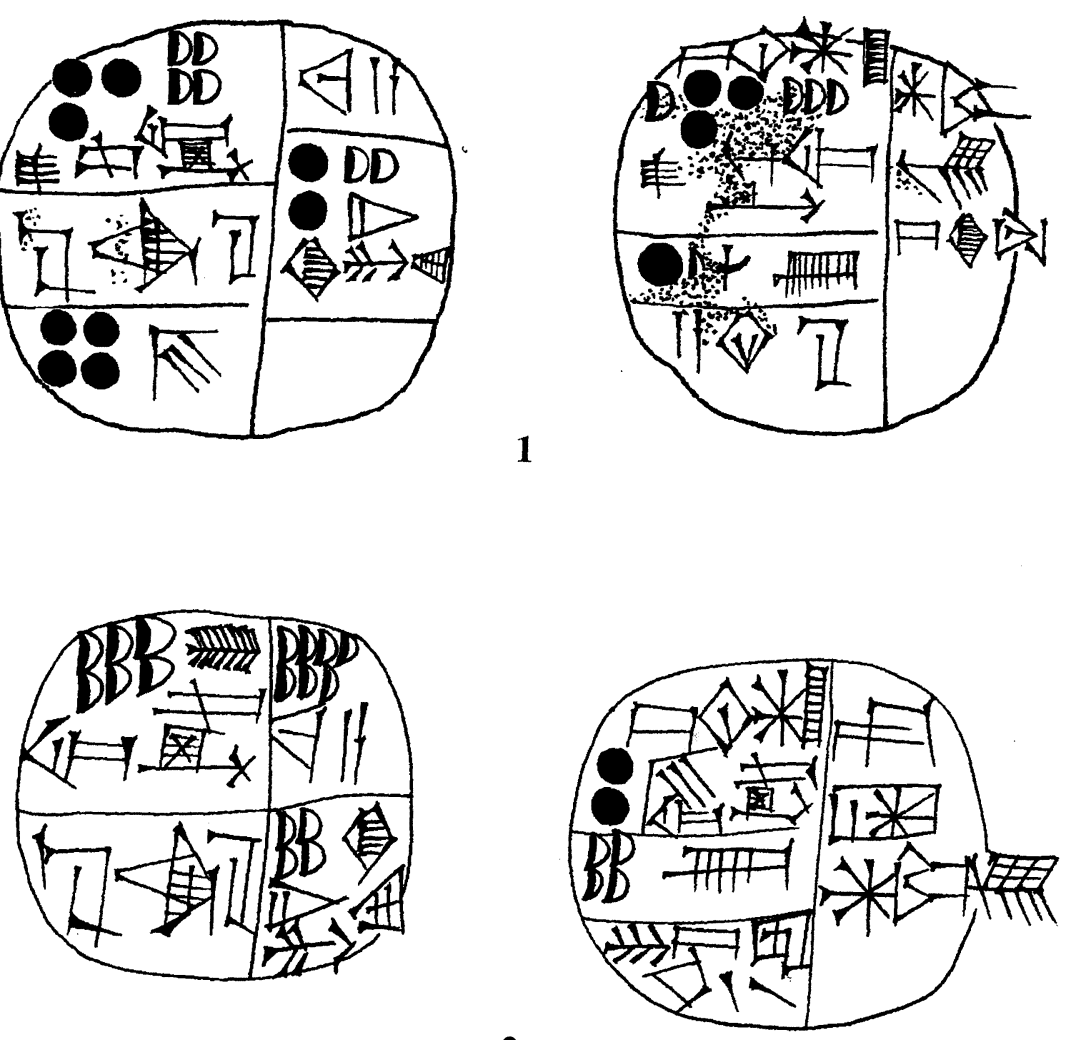

2

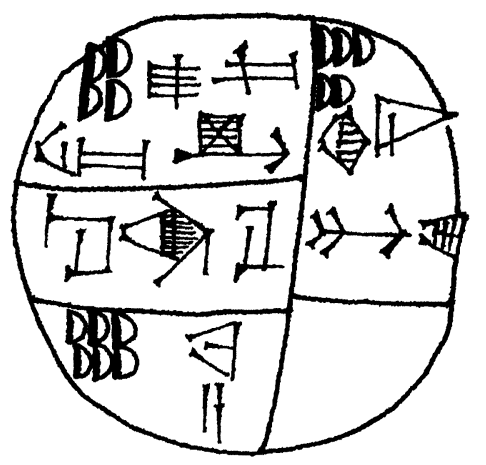

3

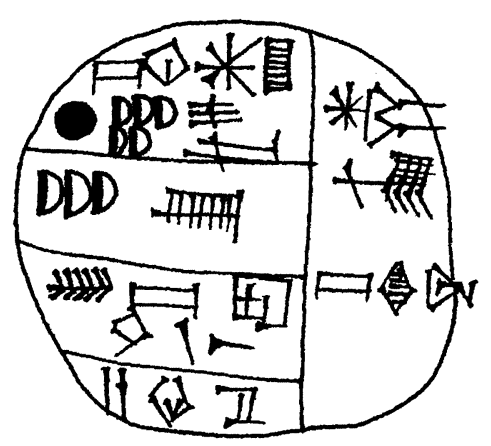




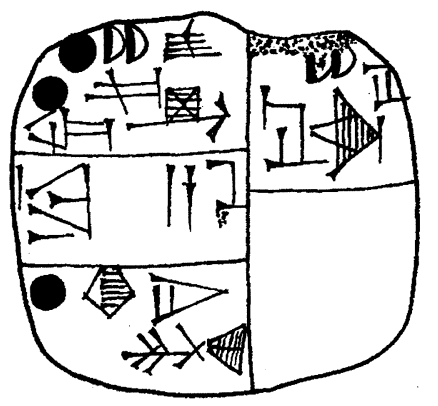

4
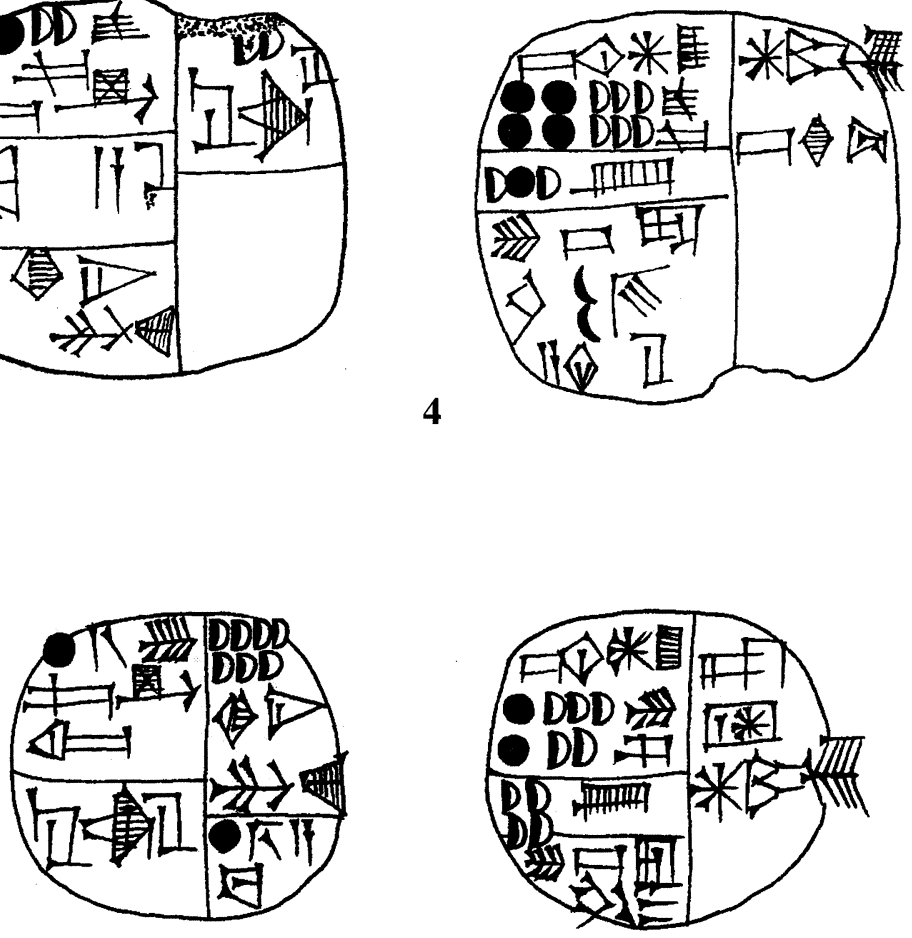

5
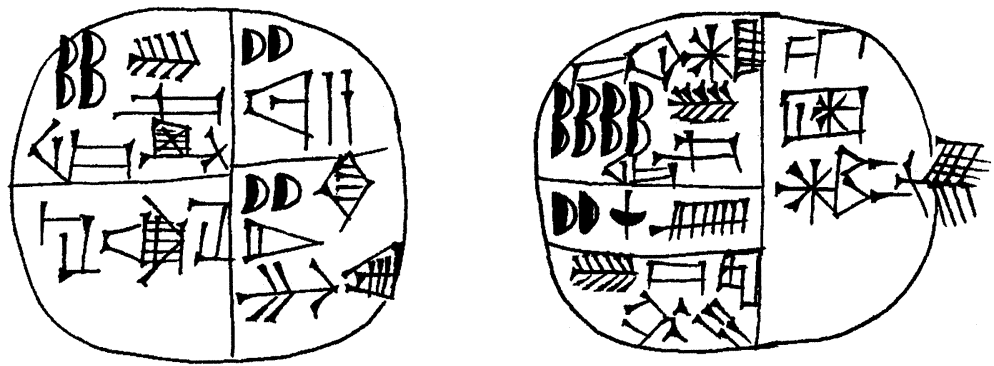

6 

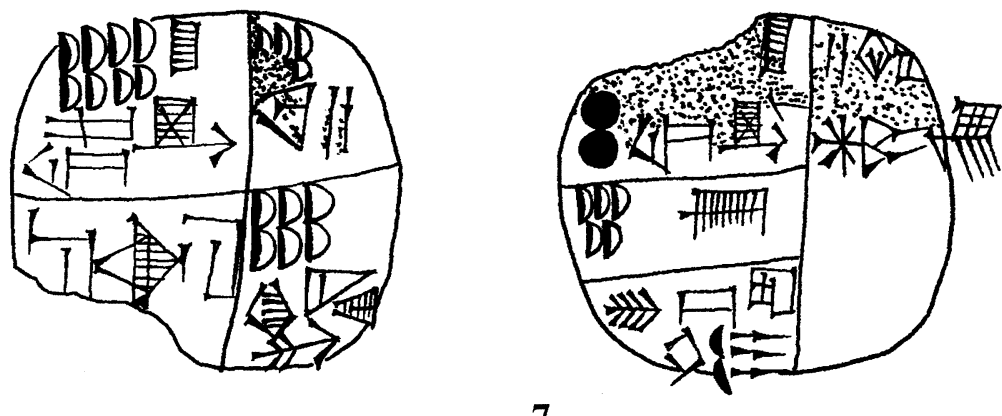

7
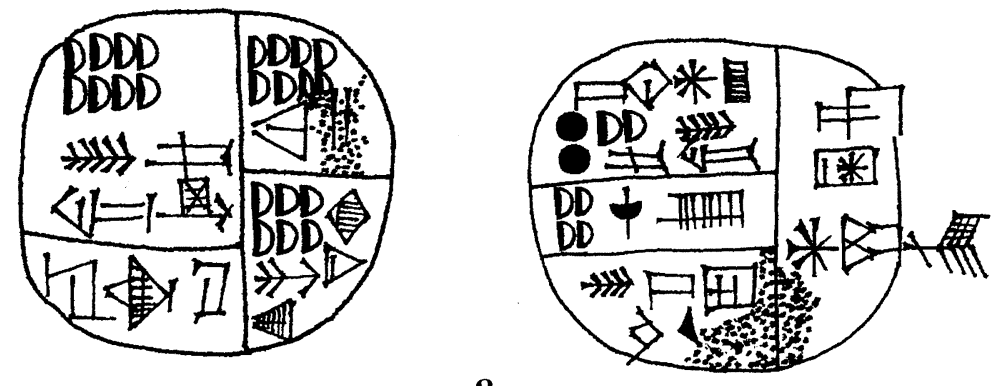

8

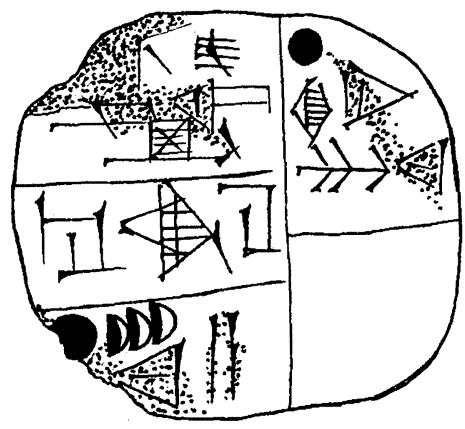

9

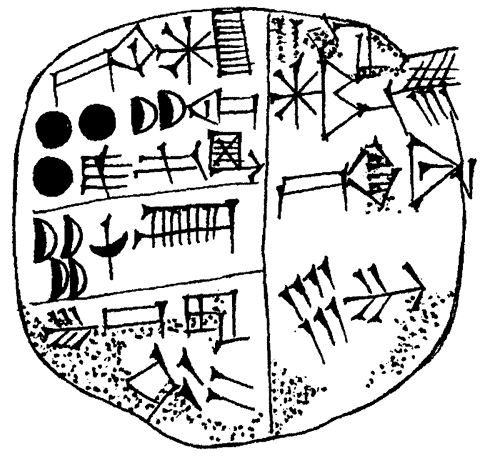




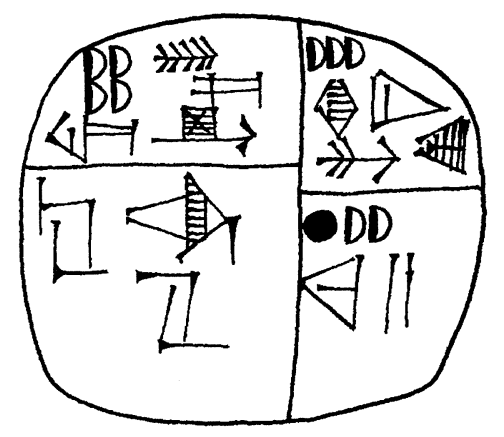

10
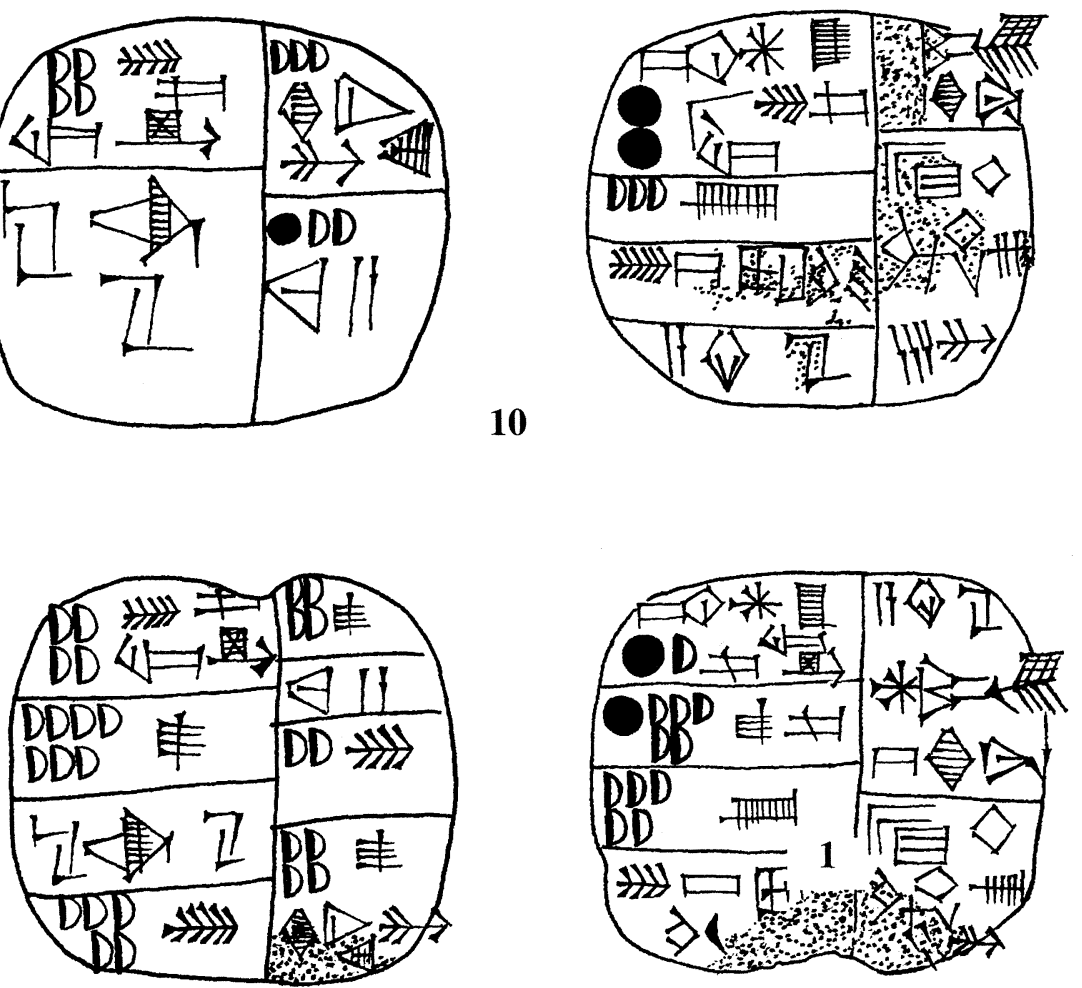

11

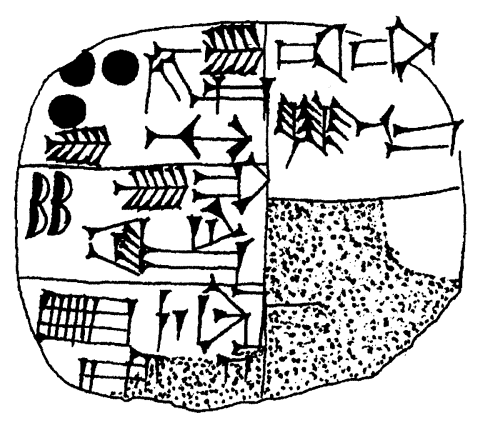

12

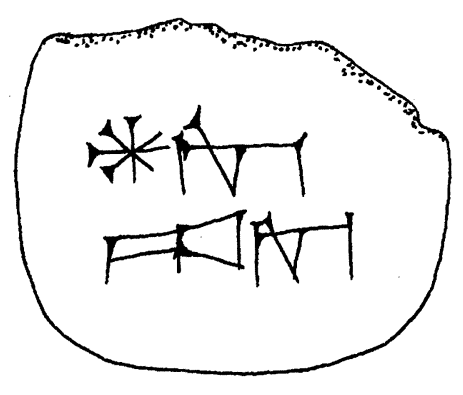




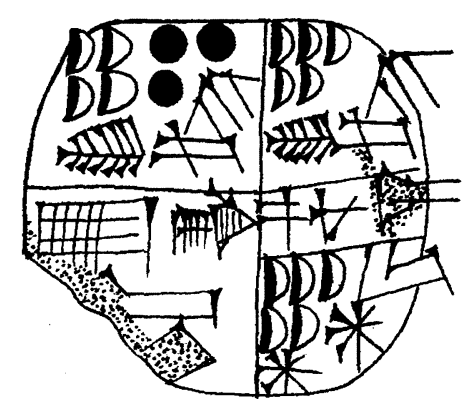

13
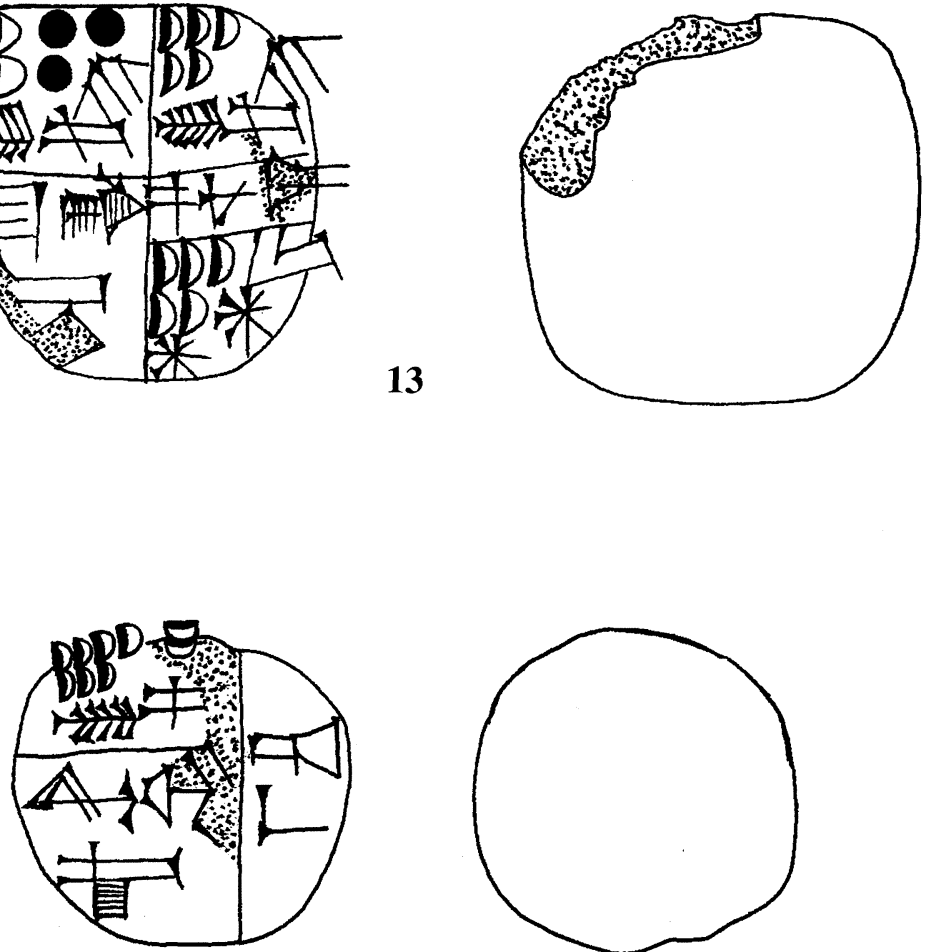

14
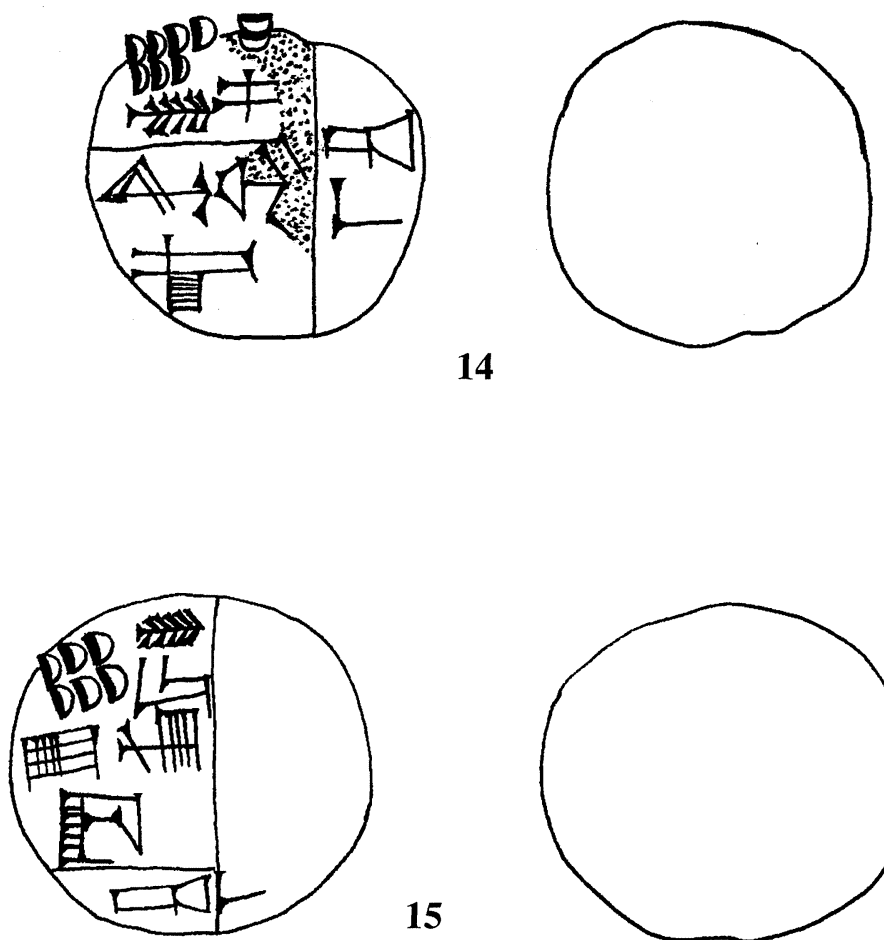

15

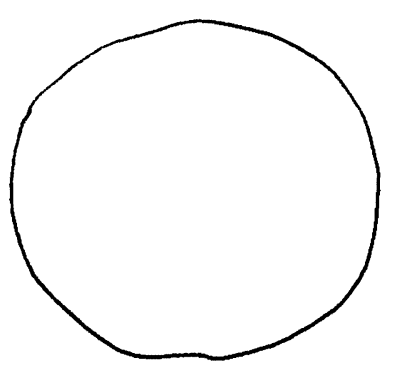



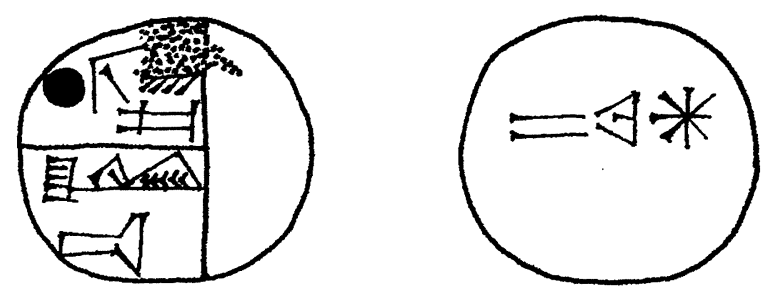

16
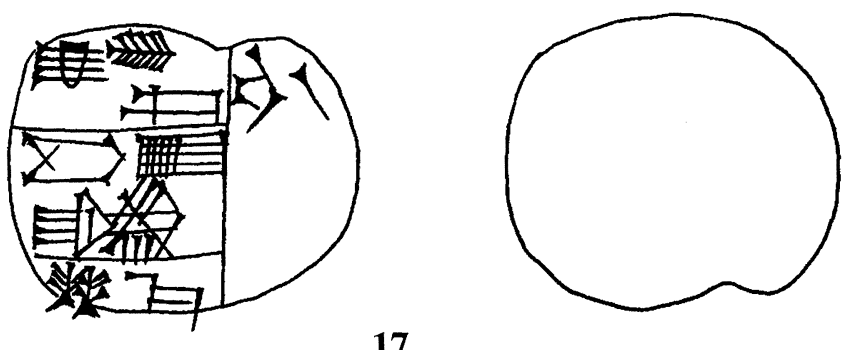

17

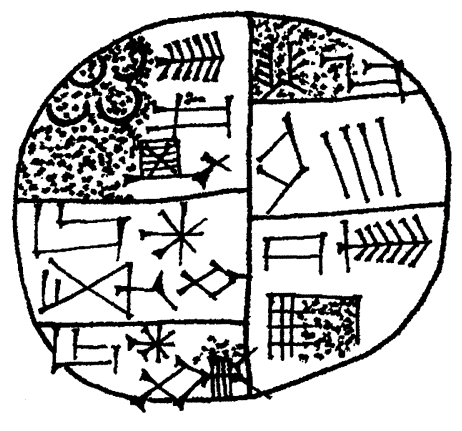

18

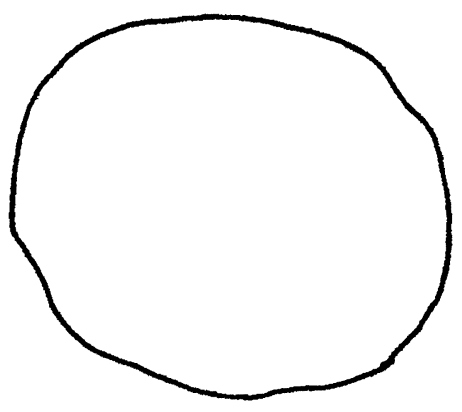




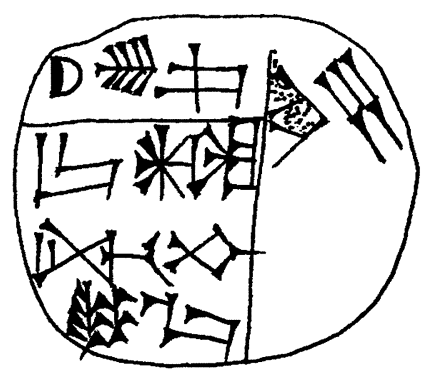

19
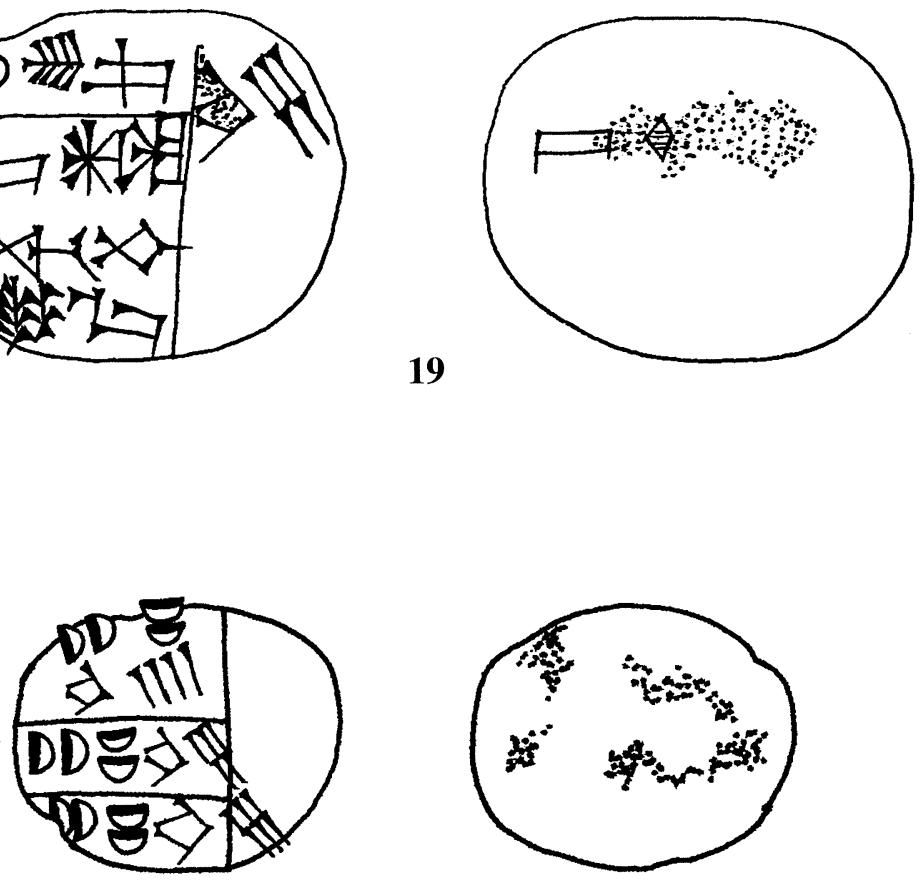

20 


\section{RESUMEN}

En este artículo se edita un pequeño archivo de veinte tablillas cuneiformes. Los textos, que proceden de la ciudad-estado de Umma-Zabala y datan del periodo Protodinástico III $b$, tratan de cereales y de actividades agrícolas.

Palabras Clave: Textos cuneiformes, Umma-Zabala, periodo Protodinástico III b.

\section{SUMMARY}

In the present paper a small archive of twenty cuneiform tablets is edited. The texts, coming from the city-state of Umma-Zabala and dating to the Early Dynastic III b period, deal with cereals and agricultural activities.

KEYWORDS: Cuneiform texts, Umma-Zabala, Early Dynastic III b period. 